\title{
On the Interpretation of Phonological Primes ${ }^{1}$
}

Gaberell Drachman

\section{Introduction.}

The core of an empirical science may be said to consist in the quest for two kinds of principle. The first, that of prediction (Hempel 1953), 2 is clearly insufficient in itself, as witness the ability of the Babylonians to predict the eclipse of the moon, but apparently without the support of any serious speculation on the nature of eclipses (Cf. Toulmin 1961; Hanson 1971). The second, that of explanation, surely includes the first (contra HempelOppenheim 1948; cf. Scheffler 1957),3 since explanation may normally allow prediction while the reverse is not necessarily true.

1.1. To gain precision, scope, and a high possibility of confirmation, sciences are codified as systems of concepts. But the requirement for empirical content implies a connection between these concepts and the world of experience. At least three kinds of connectivity have been postulated.

In the first (call it strong empiricism, even positivism), concepts are defined solely in terms of an observational vocabulary, whether sensory or instrumental. But in such an analysis, what would be the status of such a concept as latent learning, or any other predisposition to behavior, whether human or in the world of physics? Does magnetism disappear in the absence of an attractable object, or are the tendencies of the vocal tract absent when we are not in the act of speech?

1.2. The second kind of connectivity (call it liberalized empiricism) handles concepts--especially these troublesome disposition termsin terms of reduction sentences, some of which prove to contain empirical laws and are thus immediately verifiable by experiment. Thus, a reductionist analysis of the disposition term 'assimilation' in phonology might contain the sentence "If a speech organ A is to be in a position $Y$, then it will move towards that position even while it is still taking up its prior position $X^{\prime \prime}$, where $A, X$ and $Y$ are clearly definable in terms of bulk, inertia, etc., for the organ concerned.

1.3. But there are also sets of concepts (such as mass, energy) the preferred treatment of which presupposes yet a third aim for science, viz., the construction of systems of nomological relations quite abstracted from even casual explanation (Cf. Scheffler, 1957), 
as a map is neutral so far as particular routes are concerned (Toulmin 1953). In such a view, the primes of a science are best hanaled within formal systems, i.e., as uninterpreted concept systems, accompanied by appropriate sets of postulates (definitions and assumptions). Such systems, if one requires of them only inner consistency, and no assertion be made about the properties and relations of objects in the external world, have been termed 'Euclidean' (Boltzmann 1905), where the requirement of internal consistency corresponds to Herz' (1899) criterion of 'logical permissibility'.

But in fact the 'over-kill' power and hazardous temptations available within such purely formal systems are by most scientists at least tempered by the precuation of considering the (empirically) desired interpretation at the point of choosing the primes themselves; and indeed such a version of the formal system approach seems to underlie the analysis of the phonology of English in Chomsky and Halle (1968). That the power of the 'system' has, despite the precaution mentioned, not been sufficiently checked, will appear.

1.4. If the interpretation of a prime involves assigning to it some empirical content, this may clearly be done in any of at least three ways. We may interpret primes directly, as in a positivistic science. We may, as in biology, give content to primes indirectly by interpretation of a defined term such as 'cell fusion'. Or we may, alternatively, validify our primes by appeal to the hypotheses forming part of our Postulates: in this case, what are at length tested are the deductions ve make from our hypotheses or assumptions. 4

\section{Phonology as a scientific theory.}

It is illuminating to consider the advantages and limitations of constructing phonology in terms of such a formal theory of primes and postulates. I shall here take "The Sound Pattern of English" (hereafter SPE) as a representative case in point, and examine some of its primes and essumptions.

2.1. The primes of the system here are entities such as Distinctive Feature, Boundary, Rule of Phonolopy, etc. The postulates include definitions; such are the definitions of segment, formative, derivation, etc. They also include assumptions, of which the following are examples: (1) that the sets of Distinctive Features (DFs), Boundaries, Formatives, etc., are finite, (2) that the inputs to the phonological rules are syntactically-motivated and labelled surface-strings of (underlvingly-shaped) formatives and abstract formatives, plus boundary markers, (3) that phonological rules may modify, permute, delete, or add segments of formative representations, (4) that rules of phonology are (if ordered) linearly applied, and cannot re-apply to their own output in the same applicational cycle, (5) that all the phonological rules have equal status and are equally vell motivated, and (G) that all phonological rules represent competence (i.e., knowledge, or relations, rather than behavior), but that fast speech, coarticulations, etc., are matters of performance. 
2.2. The interpretation of primes is in SPE carried out in terms of performance requirements. Two sample problems may be raised here. First, systemic considerations in fact interrupt the flowchart by which one predicts real-time outputs. Thus, adjustment of formatives (e.g., 'sing + past' $+s^{*} n g$, and 'mend + past + mend+d) must of course precede the assignment of pluses or minuses for the individual DFs within segments. But this assignment constitutes the use of DFs in their systemic or classificatory (i.e., uninterpreted) function, which thus follows a performance requirement (the so-called 'adjustment' of formatives).

Second, it is dfficult to decide in principle, when 'interpretation' involves integers on DFs, and when further DFs are in fact required. A growing range of processes, and interactions between processes, has been described. It is even seen that processes may appear to be self-contradictory, with a given environment apparently provoking opposite effects in different languages, or even different periods of the same language. For example, the vowels in the neighborhood of nasals are sometimes raises, sometimes lowered; similarly, where $/ \mathrm{h} /$ is usually (i.e., most frequently attested as) a vowel-lowerer, there are cases (e.g., in Classical Greek, see Malikouti-Drachman 1972) where it seems to behave as a vowel-raiser.

Now while the explanations for such apparent contradictions are perhaps all to be sought in considerations of physiology and perception, it is puzzling whether the details of the explanations, whenever these come to light, need in fact to be built directly into the rules themselves, viz., as additional DFs. Pace Vennemann's (1971) explication of back-vowel lowering by coronals as relating to the backwards-slope of the body of the tongue, is a DF 'tongue-slope' to be added? Similarly, what DFs would correspond to the putative explanation for the Greek case above, that $/ \mathrm{h} /$ here probably partly unvoices the preceding vowel, with consequent (perceptual-based) raising?

What is not clear is whether, as the full possibilities of the vocal tract are disclosed and many further processes are isolated, the number of $\mathrm{DF}(\mathrm{s})$ required to state all processes in language will remain usefully finite. By way of providing for this eventuality in advance, one ought perhaps to consider the possibility of abandoning the requirement that the rule-format should itself contain the explanation. Without this requirement, the rules themselves could be stripped of all 'understood' detail, although (as well be suggested below, Sections 3,5) an important distinction is required in the way in which different rules are to be handled in this respect, as well as an important enrichment of the metatheory of phonetics.

\section{On the equality of processes.}

One of the most important assumptions made in SPE is that all the rules postulated are of equal status and are similarly motivated. From this assumption hangs the notion, too, that the phonological component of a gramar constitutes a seamless web of rules; and it follows that linguistic significance is denied to any level between 
lexical (phonological) and phonetic (uninterpreted DF) representations. The fundamental assumption, however, is not unchallenceable, as I shall here attempt to show.

3.1. In SPE, the interpretation of DFs consists of assigning an integer to each plus sign, with the implication that the values to be assigned are in fact mutually independent from segment to segment. But there is some reason to believe that an important generalization is missed by this assumption; a generalization captured by the classical notion Basis of Articulation (hereafter, the Basis). In explicating the role of the Basis in phonology, I have claimed (Drachman 1973) that there are elements in the language specific tract stance which in fact fuarantee or exclude whole sets of processes, the Basis thus constituting a kind of casual principle. In this sense, the notion of the Basis is grounded in a very general principle in biology, which asserts that motor-systems may be pre-primed for specific activities, an interpretation which allows for innate (i.e., universal) as well as learned (i.e., languagespecific) elements to obtain in speech-priming.

Thus there prove to be global priminf elements that are probably genetic in origin, such as the re-organization of the breathing program, as well as the fact that the members of the speech-tract are pre-set at all. But there are also language-specific elements, such as the shaping and attitude of the tongue, 5 height of the larynx, height and inner tension of the velum, as well as dependency elements such as the freedom of the lips to coarticulate with a following vowel, etc. That a single element of the Basis may guarantee both positive and negative outputs (sponsoring as well as blocking processes) may be briefly illustrated from the remarks of Delattre (1953) on French, an example of which is his 'mode anterieure'. Delattre's 'mode anterieure' may be looked upon as the articulatory implementation of a single acoustical aim, the dominance of 'forward resonance'. To this end, the tract attitude includes a convexed, downard-pointing forward-draw tongue, with dominated (i.e., freely coarticulating) lip-roundinf. A number of fine phonetic facts follow from this global tongue-lips stance. These include diverse positive factors (sponsored rules) governing the true dentality of dentals--before front vowels, the tip of the toncue is actuelly behind the lower incisors--the dorsal character of $/ \mathrm{r} /$, and the proneness of vowels to nasalization. However, the very same tongue stance also guarantees negative factors (blocked rules) such as that dental obstruents never palatalize in French, even in the most casual speech.

So far as English is concerned, the Basis probably applies for the rule series at least including and following palatalization--as confirmed by the productivity of the latter in external sandhi; and conversely, the rules precedinf this series must be represented at sone higher level in the control system, or sinply as non-realtime rules. 
3.2. Faced with such considerations, the argument appears to have swing back to the claim at flrat challenged, $v 1 z$, that there is a natural break in the continuity of the SPE rule systen. However, not only is the place of the break not the same as in the SPE clatm, but it is also quite differently motivated. In particular, it is likely that a development of the theory of the Basis will support the notion that (Cf. Zwicky 2972b) there is a contInuum of processes beyond (1.e., later than) those in SPE, processes explicating the facts of fast (or, casual) speech. Such a development involves consideration of how the Basis is itself adjusted to sponsor various degrees of casualness, so that rules are phssed in or out in accordance with natural hierarchies.

It is important to note, finally, that the use of the term 'guarantee' in connection with the operation of the Basis must be refined. For it is not the case that all that the activation of the elements of the Basis is quite automatic: the Besis only. makes the operation of the appropriate processes easeful and natural-provided they are to be allowed. This is in fact only to re-affirm the very reasonable constraint that all language processes operate quite within the limits imposed by physiology.6 Thus, it need not surprise us to find many quite idiosyncratic exceptions to rules (whether by virtue of lexical, morphological or even syntactic constraints--cf. Zwicky 1972b), for any given language, even where these rules seem to concern very fine phonetics.

\section{Phonology as a non-continuous structure.}

The hypothesis that the phonological component is in fact a two-level one may in turn be tested against derived hypotheses. This assay is made partially below, with the aid of three such hypotheses.

4.1. The first derived hypothesis might be that the 'output' rules truly constitute a real-time component of the phonology, in fact an integral part of the performance component--although of course 'performance component' is not to be thought of solely in terms of mechanical organs, but rather includes also some of the 'upstream' apparatus of the neurophysiological control system.

The real-time requirement, though ill-understood in fine, may be grossly correlated with (1) brain synapse-times, (2) impulsevelocities in cranial nerves, (3) muscle-contraction times, and (4) speech-tract inertial constants. An estimate such as that of Reich (1968) may prove to be over-sanguine, failing as it does to take account of factors (3) and (4) above; but notice that this failure affects the total number of possible processes per second (and thus his figure of 200 processes per syllable) only by cutting back somewhat the number of muscle-contraction type of processes, not the number of brain processes.

In the end, it remains unnecessary to assume (pace Wickelgren 1969) that some 104-6 language-specific output segments are 'stored' as context-sensitive units: there does seem to be time for these to be generated by rules that are fully supported by the Basis. 
4.2. The present evidence for a divided phonology comes in the first place from the Basis, as outlined above. It seems supported too by evidence from slips of the tongue and children's secret languages, as perhaps also from some kinds of data from aphasia. But there is also a derived hypothesis for rule-systems, a hypothesis which at the same time tests the main hypothesis and acts as a touch-stone for the well-formedness of rule systems themselves.

This second derived hypothesis is that, if the rules of a phonology fall into two groups, of upper and lower-brain (hereafter, upper and lower) ruies, 7 then it ought never to be the case (i.e., in a particular set of rules) that an 'upper' rule follows, or even operates simultaneously with a 'lower' one. An interesting case in point is provided by the treatment in Kiparsky (1971) and Koutsoudas (1971) of data from certain Swiss German dialects first analyzed in Kiparsky (1968).

In brief, Kiparsky describes the relation between certain forms in the (conservative) Schaffhausen and (innovative) Kesswill dialects as illustrating a putative universal of linguistic change, viz., that the innovating dialect minimizes 'bleeding' by adopting 'counterbleeding', here for the pair of rules (here given in the 'conservative' bleeding order)

1. Umlaut, for Plural

2. Lowering of mid-back vowel before a Coronal

Noting that Unlaut is for German generally an 'upper' rule, it is clear that Kiparsky's putative universal of change presents us with a counter-case to the derived hypothesis above, viz., that 'upper' rules should never follow 'lower' ones.

It must thus be of interest to consider any re-analysis that avoids this conclusion, especially if the analysis shows other intrinsically interesting features. A candidate re-analysis is thus that of Koutsoudas, who holds that in fact the extrinsic ordering illustrated in Kiparsky's analysis of the conservative dialect is unnecessary. 8 'ihe proffered alternative analysis assumes that for both dialects simultaneous ordering is possible, with the addition of a context-free condition (for the conservative dialect) that front rounded vowels cannot remain low. 9

How from the point of view of the metatheoretical constraint proposed on the ordering of 'upper' and 'lower' rules, this analysis is still not quite satisfactory, in that one each of such rules are held to operate simultaneously--vhile Umlaut is certainly an 'upper' rule, mid-back-vowel lowering certainly seems a 'lower' one. There seems no way out of the dilemma at the moment save to suppose that, since both rules cannot be considered 'lover', perhaps they are both 'upper'; and it remains unclear whether the analysis or the principle is what is at fault. Certainly, Kiparsky himself fives reasons for tempering the absoluteness of his principle of llleedingireference: in particular, he cites the need to balance arainst it factors such as the possibly opposing principle of phonotactic naturalness. 
This case 1llustrates well the problem of the concept 'purpose' in scientific anelys1s. Whereas ethologists have in many cases establiohed putative 'motivation' for certaln innete functions in nature (e.g., the coloration of butterfly wings, the complex 'dance' of the triple-spined stickle-back), we lack adequate criteria that will exable us to extend the principle to linguistica without hazard. On the one hand, the causal principle ouggested for the Basis seems a genuine example of a substantive analogy (In the sense of Hesse 1966); it suggests exactly what mechanism is at play in an experimentally verifiable situation. On the other, the principle of 'purpose' in language-change lacks this kind of veriflability and involves the delicate welghing of contradictory forces.10

4.3. A third derived hypothesis for a two-level theory of phonology concerns the theory of learning; there ought to be correlated at the level of neurological mechanisms, to the distinction between 'lower' and 'upper' rule and the way in which they are respectively 'acquired" by the child.

According to Piaget (1926, and passim), the child develops by the two complementary processes of assimilation and accommodation: new data are first assimilated to existing schemata, but these same schemata are later accomodated under the pressure of fresh data. In applying such notions to the data for the acquisition of phonology, one must first enquire, how the very first achemata themselves are acquired by the growing child.

The strongest hypothesis concerning the earliest schemata is that these are in fact innate, a hypothesis first proposed for phonology by stampe (1969). On that basis (compare the debate in Drachman 1972a) some further assumptions one might make are: (1) that the child brings as 'given' a whole train of 'live' processes-corresponding to the natural predispositions of the human vocal tract, (2) that these processes in effect 'funnel' the diversity of inputs into a narrow range of possible outputs, perhaps by lateral motor inhibition ( $\mathrm{CP}$. Békesy 1967 , on sensory inhibition), (3) that for a given formative, only the 'final' outcome of the process-train is a suitable input to the cranial-nerve command-system for moving the members of the vocal tract, and (4) that each individual process 'hunts' across a given formative before giving place to the following process.

Thus, for the acquisition model, the incoming data may certainly be seen as assimilating to existing schemata (here, innate), as is clear from the poverty-stricken output of the chlld for the earliest language stages. And it is equally clear that the innate schemata are themselves slowly accommodated to the pressure of the data. The child makes strong and creative efforts (Cf. Drachman 1971, 1972b) to undo the homonymy created by the inevitable operation of the funneling processes, and match his output to the input. Later, the relations he discovers between stylistic variants (casual, supercorrect, etc.), and in paradigm alternations, etc., are slowly subsumed under ever more abstract representations, with correspondingly longer sets of rules for the derivation of complementary outputs. 
There are thus at least three kinds of learning (accommodation) to be accounted for, viz., (1) that tract-command improves, so that the funnelling effects of natural (inhibition?) processes is slowly overcome (de-inhibition?), (2) that processes, or even trains of processes, may be tagged with information pertaining to morphology or syntax--a tagging that may also apply to individual lexical items viz-a-viz processes, and (3) that representations may become more abstract, so that processes must apparently be added to trains: depending on the degree of abstraction of the re-modelled representation, these may be 'lower' processes, re-activated--as in the case of the very early limited abstractization of surface phonetics (cf. Stampe 1968); or they may be 'upper' processes, if the child indeed takes advantage of every regularity in the data to 'solve' paradigm alternations such as Umlaut pluralization.

Whitaker (1971a) first sugfested a possible connection betreen the learning abilities of the child, and the distinction made by biophysicist Jakobson (1969) between specific and non-specific neuronal connections in the brain. On the basis of research performed largely on fish and amphibian eyes, Jakobson supposed the 'wiring' of the brain to proceed in two major stages. For innate behavior (that not requiring environmental triggering) the basic wiring contains invariant connections. But for learned behavior (that requiring environmental triggering), he postulated the mediation of connections between unconstrained (non-specific) neurons, i.e., neurons requiring functional validification.

In terms of the assumption concerning 'lower' processes, we might assume that the fundamental train of these processes is laid dow in terms of specific (invariant) neuronal connections, to be trigfered (perhaps by a hormone) at some internally-predetermined point in time. For the learned (or, 'upper') processes, Whitaker has, reasonably, implicated the non-specific (unconstrained) neurons; these, he says, are activated at about 1 year, and constitute "the basis for native language habits".

Two problems immediately arise. First, it must be explained how, if innate processes depend upon invariant connections, they can be suppressed or even modified at all. The solution to this apparent paradox lies in viewing processes as a function not of individual connections but rather of networks of such connections. Network-internal excitability is what is indeed modifiable: it may be modified directly, by the intervention of selective-action hormones on particular neurons; or indirectly, throurh changes induced at synapses or neuron connections (Jakobson 1967).

The second problem is, why, if they are mediated by non-specific or modifiable neurons, do 'upper' processes cease to be modifiable as soon as they are learned? In the end, the functional analory with the eyes of fish and amphibia may prove to be unproductive--for it is almost the case that we should prefer to have the system develop in the opposite direction, with the modifiable neurons corresponding to the modifiable processes and the unmodifiable ones corresponding to the obligatory ('upper') rules. 
5. Uninterpreted and interpreted primes.

If we assert that primes ought perhaps to partake in the system as uninterpreted elements, we restore the full power available to system-emphasizing analyses. This seems to be both the strength and the weakness in the analysis in SPE, where DFs have their ill-defined classificatory function within the phonological component, but are given their well-defined phonetic (interpretive) function at the interface with the performance component. This paper has attempted to modify this position to the extent of asserting that the interpretation must in fact come earlier, since some rules (those co-opted, by the Basis of Articulation) clearly operate in real time, and thus constitute a part of the real-time or performance component.

On the one hand, some cases of the SPE use of devices such as bracketing will automatically be avoided. For example, the single (bracketed) rule for vowel Tensingll is in the present framework clearly two separate rules. The first is 'upper' and applies to Lax vowels preceding vowels, as in 'various: varlety'--where the rule is obviously ordered before the Vowel Shift. But the second, applying to Lax vowels word-finally, as in 'hindu', is clearly 'lower'--as witness the treatment of French words with final Lax vowels, e.g., Englishized 'coupé'.

On the other hand, rules understood as 'upper' in the prsent framework are st1ll vulnerable to such 'over-kill' devices as the alpha-convention-for example, as applied in the rules for the Vowel Shift in SPE.12

One solution to the problem of the over-power in the formal analysis might at first sight be to impose the interpretation requirement on all the rules (and rule schemata), for the whole phonological component homogeneously. But this carries with it the quite unwarranted implication that the 'upper' rules are in fact all properly motivated in a synchronic phonolofy. This is a proposition difficult to reconcile with what has been held concerning the function of the Basis; for it implies that, as changes in the Basis are historically to be associated with (even sometimes to be held responsible for) sound changes, the synchronic phonology must necessarily recapitulate all such changes in the Basis to ensure that the rules operate in plausible fashion. In the extreme case, each rule might require its own individual statement concerning the Basis, a statement to be modified for the following rule, etc.

In the end, since this last can hardly be a plausible synchronic solution, we are again left with uninterpreted primes so far as the 'upper' rules are concerned; and constraints on the abuse of the excessive power of rule schemata thus unavoidably re-introduced must be sought elsewhere--perhaps psycholinguistic experiments on young children might elucidate which schemata are reasonably operative during acquisition, as might also childhood aphasia and psychosis studies.

On the other hand, the rule-schemata for at least the 'lower' rules can be radically simplified without loss of plausibility. If the explanations are relegated to the metatheory, as suggested above 
(section 2), the application or non-application of whole constellations of rules can be predicted from consideration of the Basis for the language concerned, as plausible or implausible: it will remain to state whether or not the language in fact allows these rules to operate universally or whether idiosyncratic constraints are to obtain.

\section{Summary and Conclusions.}

1. Three views of scientific theory are outlined, from the point of view of the way in which they handle the interpretation of primes; strong empiriciam, liberalized empiricism or reductionism, and the use of formal systems of uninterpreted primes.

2. 'ine third of these is illustrated with a particularly welldeveloped example, that of SPE. But some of the assumptions constituting part of the postulates accompanying these primes are examined, on the grounds that such testing is necessary if the primes themselves are to be validated.

3. Especially challenged is the assurption that the phonological component of a grammar is a seamless web of equally well motivated rules, and that these may be followed by the Interpretation as part of the description of Performance. It is sugfested instead that the real-time interpretation of DFs must obtain at latest before the end of the SPE system, and in particular from the point at which the Basis of Articulation guarantees the low-level outputs sanctioned in the language concerned.

4. 'Three subsidiary hypotheses derived from the assumption of: a two-part phonology are then examined: (1) the time-requirement, which makes quite feasible a real-time phonology, at least for the 'Iower' rules, (2) the implications for phonological chanse, one of which is the constraint that innovation oucht never to re-order an 'upper' rule to follow a 'lower' one, and (3) speculations from neurology', which perhaps support the cognitive (piagetian) model for the acquisition of such a prionology.

5. Finally, since it seems unreasonable to require interpretability over the whole rule-system, the problem is considered, how to constrain the over-powerful devices apparently allowable within the formal (i.e., uninterpreted) part of the phonology. It will hopefully prove possible to apply psycholinguistic tests to determine the reasonableness of particular putative rule-schemata. 


\section{Footnotes}

1. Th1s paper will appear in the Proceedings, VII LInguistisches Kolloquium (N1jmegen) 1972.

2. Except where otherwise indicated, the framework of the argument of Section 1 is essentially a paraphrase of Hempel (1953).

3. The temporal asymmetry between explanation and prediction discussed in Scheffler (1957) suggests that explanation is the more significant. But Scheffler's argument is really that nelther prediction nor explanation plays a central role in science.

4. Note that the interpretation will still unavoidably contain disposition terms. This caveat, so far as phonology is concerned, corresponds to the notion that rules describe not inevitabilities but predispositions to processes--a notion to be considered in Section 3.2. Of such predispositions, the notion 'degree of probability of behavior' must correlate with what is understood of hierarchles of environment (cf. Zwicky 1972b).

5. Shaping refers to the concave/convex/flat displacement of the tongue; attitude, to its vertical and/or horizontal displacement.

6. A claim difficult to substantiate in that it is not clear how to define fastest, or most casual, speech (Zwicky 1972a); defined in terms of the speaker's intention to communicate ( $C r$. Dressler 1972), even blurted speech must be included. And even blurted speech is, in appropriate circumstances, comprehensible to an involved hearer.

On the other hand, experiments in Kozhevnikov and Chistovich (1965) suggest that the degradation of fast speech forms is not the result simply of moving the articulators more quickly: rather, it seems that normal speech targets are not reached, since the articulators actually move more slowly. But this is to say that there are in fact (separate) intended targets appropriate to casual speech. This is a conclusion supported by the (mentalistic degree of stress) constraint on vowel-reduction for forms like deportation and relaxation, as against the corresponding forms like demonstration, devastation (SPE); and the (even nearer to the output) constraints on co-articulation, say, for Russian (Ohman 1966).

7. Cf., from aphasia studies, Whitaker's (1971b) 'central' rs. 'peripheral' rules. Also Cf. Stampe's parallel distinction 'dead' vs. 'Iive' (Seminar, Winter 1972 at Ohio State University) rules.

8. Koutsoudas is concerned with the general problem of extrinsic ordering in linguistic analysis. The evidence from child-development has been tentatively remarked upon (Drachman 1972a) briefly, it seems that phenomens resembling extrinsic (i.e., non-feeding) ordering of processes may arise as artifacts of the language acquisition process.

Note also that one case not discussed in Koutsoudas, that of optional mutual bleeding, creates a special problem. In the pair of utterances (e.g.) [fa hím] [fər f́m], 'for him', r-loss and h-loss are mutually bleeding. But it is probably the case that, even in R.P., the first version is more formal than the second; thus we must mark the orders for style, since the more casual form is not derived, as is normally the case, from the more formal one. 
9. The apparent re-ordering in the innovating dialect consists, in this framework, simply in the removal of the constraint on height for front rounded vowels.

10. A parallel collision of criteria is exemplified in language acquisition. On the one hand, the child's motivation can reasonably be assumed to be to reproduce as much information as possible. On the other, the principle 'do what is easiest' dominates at least the early stages, with resultant truncated syntax and degraded formative-shapes. When, later, the two principles collide, the former is (at least sporadically) the stronger, as witness the various strategies including (e.g.) the 'use' of long-distance assimilation processes (Drachman 1972b).

11. McCarley (1972) first pointed to the illegitimate use of bracketing in this case, on the grounds that it implied an ordering constraint on one part of a rule, which in fact applied only to the other part.

12. Many re-interpretations have been attempted, as a result of widespread dissatisfaction with the use of the alpha-convention in this case. The latest of these is perhaps Stampe's (1972).

\section{Bibliography}

Békeśy, G. von. 1967. Sensory Inhibition. Princeton. Boltzmann, I. 1905. Populäre Schriften. Leipzig. Also in A. Dento and S. Morgenbesser, eds., Philosophy of Science.

Chomsky, iN., and M. Halle. 1968. The Sound Pattern of English. ivew York.

Delattre, P. 1953. Les modes phonétiques du francais. The French Review XXVII.1.

Drachman, G. 1971. Strategies in the acquisition of phonology. To appear in the Proceedings of the Urbana Conference on Phonolory. Also see pp. 83-98 in this issue. - 1972a. On the notion 'Rule of Phonology'. Proceedines of the 1lth International Congress of Linguistics (Bologna). See 134-145. - 1972b. Generative phonology and child language acquisition. To appear in the Axten 2, Int. Phon. Tagung (Wien). Also see pp. 146-160in this issue. - 1973. Phonology and the basis of articulation. Sprache 19. Also see pp. $115-133$ in this issue.

Dressler, H. U. 1972. Methodisches zu Allegro-Regeln. Akten 2, Int. Phon. Tagung (Wien).

Hanson, N. R. 1971. Observation and Explanation. London.

Hempel, C. G. 1953. Methods of concept formation in science. Int. Encyc. of Unified Science, Chicago.

liempel, C. G., and P. Oppenheim. 1948. Studies in the logic of explanation. Philosophy of Science 15.

Herz, 11. 1899. The Principles of Mechanics. (translated from German). London.

Hess, M. B. 1966. Models and Analopies in Science. U, of Notre Dame. 
Jakobson, M. 1967. Starting points for research in the ontogeny of behavior. Major Problems in Developmentel Blology. New York. - 1969. Development of specific neuronal connections. Science 163.

Kiparsky, P. 1968. Linguistic universals and linguistic change. $E$. Bach and R. Harms, eds., Universals in Linguistic Theory. New York. - 1971. Historical linguistics. W. Dingwall, ed., A Survey of Linguistic Sclence. University of Maryland.

Koutsoudas, A., Gerald Sanders, and Craig Noll. 1971. On the application of phonological rules. Repro. by Indiana Univ. Linguistics Club.

Kozhevnikov, W. A., and L. A. Chistovich. 1965. Speech: Articulation and Perception. U.S. Dept. of Commerce, JPRS.

Lashley, K. S. 1951. The problem of serial order in behavior. L. A. Jeffress, ed., Cerebral Mechanisms of Behavior. New York. Malikouti-Drachman, A. 1972. A controversial rule in Greek. Paper read at the Winter Meeting of the Linguistic Society of America.

McCawley, J. D. 1972. Review of The Sound Pattern of English by N. Chomsky and M. Halle (1968). To appear in IJAL

öhman, S. E. G. 1966. Coarticulation in VCV utterances: spectrographic measurements. JASA 39.1

Piaget, J. 1926. The Language and Thought of the Child. London.

Reich, P. 1968. Competence, performance, and relational networks. Linguistic Automation Profect Report.

Scheffler, I. 1957. Explanation prediction and abstraction. Brit. Journal of Phil. Sci. VII.28.

Stampe, D. L. 1968. Yes, Virginia... Paper read at the 4th Regional Meeting of the Chicago Linguistic Society. (unpublished). - 1969. The acquisition of phonetic representations. Papers from the Fifth Regional Meeting of the Chicago Linguistic Society.

- 1972. On the natural history of aiphthongs. Papers from the 8th Regional Meeting of the Chicago Linguistic Society.

Toulmin, S. 1953. The Philosophy of Science. London. . 1961. Foresight and Understanding. New York,

Vennemann, T. 1971. The interpretation of phonological features in assimilation rules. Working Papers in Phonetics 19, UCLA.

Whitaker, H. 1971a. The innateness hypothesis. Paper presented at the Summer LSA Institute, Buffalo. . 1971b. The representation of language in the human mind. Edmonton, Alberta.

Wickelgren, W. A. 1969. Context-sensitive coding, associative memory, and serial order in (speech) behavior. Psych. Rev. 76.

Zwicky, A. M. 1972a. On casual speech. Papers from the 8 th Regional Meeting of the Chicago Linguistic Society.

1972b. Note on a phonological hierarchy in English. R. P. Stockwell, ed., Historicel Linguistics in the Perspective of Transformational Theory. 